\title{
The HPV vaccination strategy: could male vaccination have a significant impact?
}

\author{
V. Brown* and K.A.J. White \\ Centre for Mathematical Biology, University of Bath, Bath BA2 7AY, UK
}

(Received 4 June 2009; final version received 13 October 2009)

\begin{abstract}
We investigate the potential success of the human papilloma virus (HPV) vaccine, taking into consideration possible waning immunity and the influence of behavioural parameters. We use a compartmental, population-level ordinary differential equation (ODE) model. We find the effective reproductive value for HPV, $R_{0}^{\mathrm{e}}$, which measures the threshold for infection outbreak in a population that is not entirely susceptible, together with infection prevalence. We study the effects of different parameters on both of these quantities. Results show that waning immunity plays a large part in allowing infection to persist. The proportion of the population not sexually active when vaccination occurs affects $R_{0}^{\mathrm{e}}$, as does the rate at which individuals become sexually active. In several cases, infection persists as a result of an infection reservoir in the male cohort. To explore this further, we introduce male vaccination and find the conditions for which vaccination of males could be considered appropriate.
\end{abstract}

Keywords: mathematical modelling; HPV; vaccination; reproductive value

\section{Introduction}

Cervical cancer is the second most common cancer worldwide, with 493,000 cases attributed to it in 2002 [2]. With the discovery that human papilloma virus (HPV) infection is a necessary precursor to the development of cervical cancer [23], hopes of reducing the prevalence of cervical cancer now lie with the development and usage of vaccinations against the HPV [1].

HPV is thought to infect about $80 \%$ of the sexually active female population at some point during their lives [7]. Of these, about 10-20\% have a persistent infection lasting more than 6 months [24]. The likelihood of developing precancerous lesions increases with long-term infection; for type HPV-16, there is a $40 \%$ chance of cervical intraepithelial neoplasia, uncontrolled cell growth within the epithelium, after infection lasting 5 or more years [1].

The prevalence of the virus is highest in young adults (16-25 years); for females in this age group, recent estimates of prevalence range from 20 [20] to $40 \%$ [11]. However, with increasing rates of breakup in long-term partnerships, there is also a growing concern that prevalence may also have a secondary peak in middle-aged cohorts [10].

Of over 120 different strains of HPV that have been identified, only about 40 are thought to infect the anogenital area, of which two strains, HPV-6 and HPV-11, cause 90\%

*Corresponding author. Email: v.1.brown@bath.ac.uk 
of genital warts [4]. Moreover, some strains are carcinogenic [23], with strains HPV-16 and HPV-18 considered to be the most prevalent, causing about $70 \%$ of all cervical cancer [23].

Two vaccines have been licensed for use: one protects against strains HPV-6, HPV-11, HPV-16 and HPV-18, while the other protects against strains HPV-16 and HPV-18 [1]. The vaccines have been widely welcomed, as testing has shown close to $100 \%$ efficacy against the two strains of HPV most linked to cervical cancer [17]. As they are so new, it is not clear how long the vaccines are expected to remain effective. Current trials show that they are still effective after 5 years, but the true duration of protection will only become evident in the next 10-20 years [16]. The UK policy for the vaccine is to introduce it for 12-13-year-old girls, with a catch-up programme for females up to the age of 18 [12], although some ethical concerns have been raised about giving the vaccine to individuals prior to their sexual debut [8]. The vaccine is already being used in several other countries, including the USA and Australia [12].

We are interested in using the 'effective' $R_{0}$ value, $R_{0}^{\mathrm{e}}$, to assess the interplay of behavioural and vaccination parameters, and how these affect the potential success of the vaccine. We also use $R_{0}^{\mathrm{e}}$ to judge the impact of including male vaccination in the vaccination policy.

This complements previous work ([13,14,18], for example) and by using a simple structure, highlights key parameter groupings that are critical in understanding how HPV could be contained in a population, by a vaccination strategy aimed either only at females prior to their sexual debut or at both males and females.

\section{Methods}

We built a compartmental model of a population, the schematic of which can be seen in Figure 1. It is a deterministic model, with parameters estimated as average values over a lifetime using age-dependent data. We do not account for heterogeneity in individual behaviour beyond classifying individuals as sexually active or inactive. In particular, there is no sexual contact or age structure. Although age structure is not included, behavioural parameter values were estimated from an age-dependent database National Survey

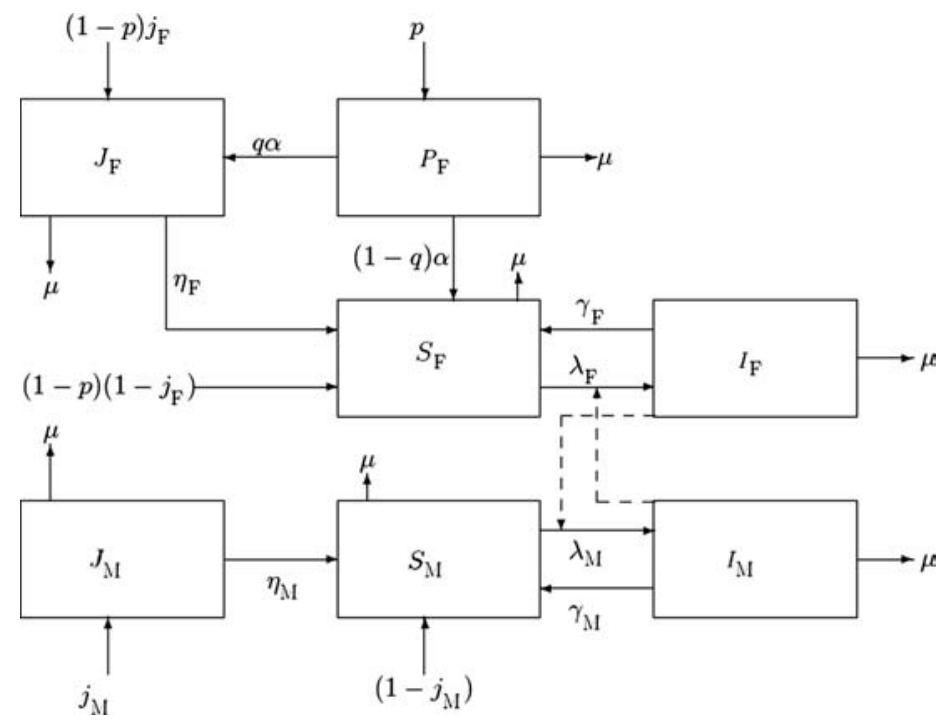

Figure 1. Schematic of model. See Table B1 for parameter definitions and text for further details. 
of Sexual Attitudes and Lifestyles II (NATSALII) [19]. As the transmission probability, $\beta$, is so high, we follow [14] and assume homogeneous mixing. Such simplifications allow us to highlight key parameter groupings such as the basic reproductive value $R_{0}$ and the effective reproductive value $R_{0}^{\mathrm{e}}$ from which the behaviour of HPV can be explored.

Considering HPV as a sexually transmitted infection (STI), we introduce two genders and study the heterosexual case. Initially, we assume that only females are vaccinated, as this is the current UK policy [12], and that vaccination takes place as individuals enter the model. We include a non-sexually active (juvenile) class to allow us to consider the interplay between waning protection and the onset of sexual activity. We present an SIS (susceptible-infected-susceptible) model, as in [15,21]. This produces a model with seven classes; protected females $P_{\mathrm{F}}$, juvenile individuals $J_{i}$, susceptible individuals (or susceptibles) $S_{i}$ and infected individuals $I_{i}$. We assume that none of the cohort entering the model is infected, thus there is no external input into the $I_{i}$ classes. Here, $i=\mathrm{F}, \mathrm{M}$ representing females and males. The parameters are as in Table B1; these estimates have a variety of published sources. The frequency-dependent force of infection $\lambda_{i}$ is set as

$$
\lambda_{i}=\frac{z \beta I_{k}}{\left(N_{k}-J_{k}\right)}
$$

where $z$ and $\beta$ are defined in Table $\mathrm{B} 1$ and $i, k=\mathrm{F}, \mathrm{M}, i \neq k$.

While the model here does not explicitly monitor the age of individuals, several parameters are directly linked to the age of vaccination. The proportion of individuals, $q$, who are not sexually active when the vaccination protection wears off and the proportion of unvaccinated individuals, $j_{i}, i=\mathrm{F}$, M, who are not sexually active are both estimated using the NATSALII database [19]. The parameter $q$ is taken to be an increasing function of $\alpha$ and was also estimated using data from [19]. Age-related gender differences are included by assuming that the rate of becoming sexually active is greater for males than females $\left(\eta_{\mathrm{M}} \geq \eta_{\mathrm{F}}\right)$ and that more males are sexually active than females when vaccination is administered $\left(j_{\mathrm{M}} \leq j_{\mathrm{F}}\right)$.

From the model, we can determine the key epidemiological parameter $R_{0}$, which measures the number of secondary infections caused by a single infection being introduced into an entirely susceptible population. Assuming that one generation of the infection is measured as female infection to female infection we obtain,

$$
R_{0}=\frac{(z \beta)^{2}}{\left(\gamma_{\mathrm{F}}+1 / \phi\right)\left(\gamma_{\mathrm{M}}+1 / \phi\right)}
$$

For standard parameters from Table B1 $\left(z=2, \beta=0.6, \phi=65, \gamma_{\mathrm{F}}=\gamma_{\mathrm{M}}=1\right)$, we estimate $R_{0} \approx 1.4$. Moreover, it is possible to calculate the effective $R_{0}$ value, $R_{0}^{\mathrm{e}}$, which takes into account the delayed sexual onset and vaccination effects [3]. For our model, $R_{0}^{\mathrm{e}}=R_{0}(1-f(p))$, where

$$
f(p)=\frac{p\left(\eta_{\mathrm{F}}+1 / \phi\right)}{\phi\left(\eta_{\mathrm{F}}+1 / \phi\right)(\alpha+1 / \phi)-\left((1-p)(\alpha+1 / \phi) j_{\mathrm{F}}+q \alpha p\right)} .
$$

This was calculated using the criteria for local stability of the disease-free steady state detailed in appendix B. $R_{0}^{\mathrm{e}}$ as defined above is comparable to the form the effective $R_{0}$ value takes when a vaccination programme is introduced into a disease situation (i.e. $R_{0}^{\mathrm{e}}=R_{0}(1-p)$ ) [9]. Eradication of infection in the population is only possible 
if $R_{0}^{\mathrm{e}}<1$, which corresponds to

$$
f(p)>1-\frac{1}{R_{0}}
$$

With the introduction of male vaccination, $R_{0}^{\mathrm{e}}$ is modified to $R_{0}^{\mathrm{e}}=R_{0}\left(1-f_{\mathrm{F}}(p)\right)$ $\left(1-f_{\mathrm{M}}(p)\right)$, where

$$
f_{i}(p)=\frac{p\left(\eta_{i}+1 / \phi\right)}{\phi\left(\eta_{i}+1 / \phi\right)(\alpha+1 / \phi)-\left((1-p)(\alpha+1 / \phi) j_{i}+q \alpha p\right)} .
$$

Initially, we specify all gender-specific parameters to be the same, which means that $R_{0}^{\mathrm{e}}=R_{0}(1-f(p))^{2}$, and then infection can only be eradicated for the condition

$$
f(p)>1-\frac{1}{\sqrt{R_{0}}} .
$$

\section{Results}

We use (3)-(6) to explore the interplay between key behavioural parameters $j_{i}$ and $\eta_{i}$ and vaccination parameters $p$ and $\alpha$ in predicting conditions under which HPV could be eradicated by an appropriate vaccination strategy.

The dependence of $f(p)$ on these parameters is shown in Figure 2, from which we see that $f(p)$ is an increasing function of $p$ but a decreasing function of both $\alpha$ and $\eta_{\mathrm{F}}$, with variation in $\alpha$ causing the greatest variation in $f(p)$. According to (4) and (6), eradication of infection can only occur if $f(p)$ is sufficiently large - we see from Figure 2 that this will only happen if either $p$ is sufficiently large or if $\eta_{\mathrm{F}}$ or $\alpha$ is sufficiently small, with small $\alpha$ giving the largest change in $f(p)$.

\subsection{Vaccination in a population already sexually active}

Setting $q=j_{i}=0$, we consider a vaccination strategy aimed at a population of sexually active individuals. This is analogous to previous work presented in [18] and gives the following results:

- For female-only vaccination

$$
R_{0}^{\mathrm{e}}=R_{0}\left(1-\frac{p}{\phi(\alpha+1 / \phi)}\right)
$$

- With female and male vaccination

$$
R_{0}^{\mathrm{e}}=R_{0}\left(1-\frac{p}{\phi(\alpha+1 / \phi)}\right)^{2}
$$

In both cases, as $\alpha$ decreases the period of vaccine efficacy increases and the proportion of the population that should be vaccinated to eradicate HPV is reduced, with this reduction more pronounced when both males and females are vaccinated. For example, the value of $p$ required when $\alpha$ is essentially lifelong $(\alpha=1 / \phi)$ is less than half of that required for $p$ when the vaccine lasts for 20 years (all other parameters being equal) for both female-only 

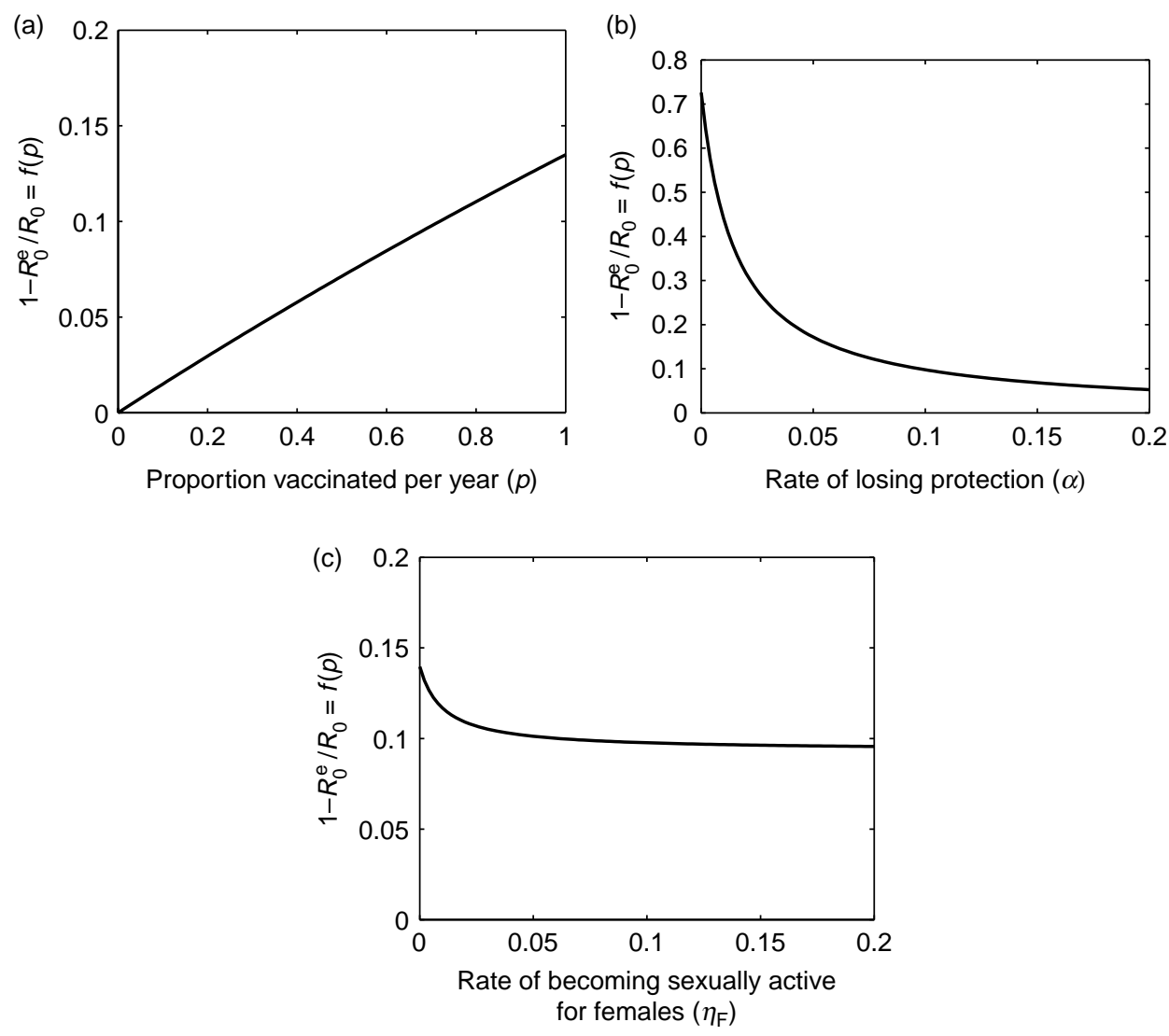

Figure 2. Three graphs showing the change in $f(p)$ as different parameters vary. Figure 2(a) shows $f(p)$ as it varies against $p, 2(\mathrm{~b})$ shows $f(p)$ against $\alpha$ and 2(c) shows $f(p)$ against $\eta_{\mathrm{F}}$. All other parameters are taken from ranges given in Table B1.

vaccination and vaccination of both sexes, although in the case of vaccination of both sexes, $R_{0}^{\mathrm{e}}$ can be driven below 1 for a lower $p$ value.

\subsection{Female-only vaccination}

Figure 3 shows how $R_{0}^{\mathrm{e}}$, for female-only vaccination as given by (7), varies with the key model parameters. Using parameters from Table B1, we see that changes to $\eta_{\mathrm{F}}$ are not able to drive infection from the population for any level of vaccination. In contrast, provided that the vaccination is sufficiently long lasting ( $\alpha$ small), then if $p$ is large enough infection may be eradicated. For example, a vaccine lasting for at least 20 years $(\alpha=0.05)$ in a population averaging two partners per year $(z=2)$, with coverage of at least $70 \%$ of the female population $p \geq 0.7$, can drive $R_{0}^{\mathrm{e}}<1$. While changes in $\eta_{\mathrm{F}}$ do not have a great impact on $R_{0}^{\mathrm{e}}$, another behavioural parameter $z$ does. In particular, a reduction of $z$ by less than $25 \%$ ( $z$ reduced from 2 to 1.6 ) results in $R_{0}^{\mathrm{e}}<1$ for a range of parameters.

Figure 4 shows infection prevalence for females and males corresponding to the $R_{0}^{\mathrm{e}}$ values shown in Figure 3. This highlights the heterogeneity in infection prevalence between the two sexes as a result of a female-only vaccination strategy. 

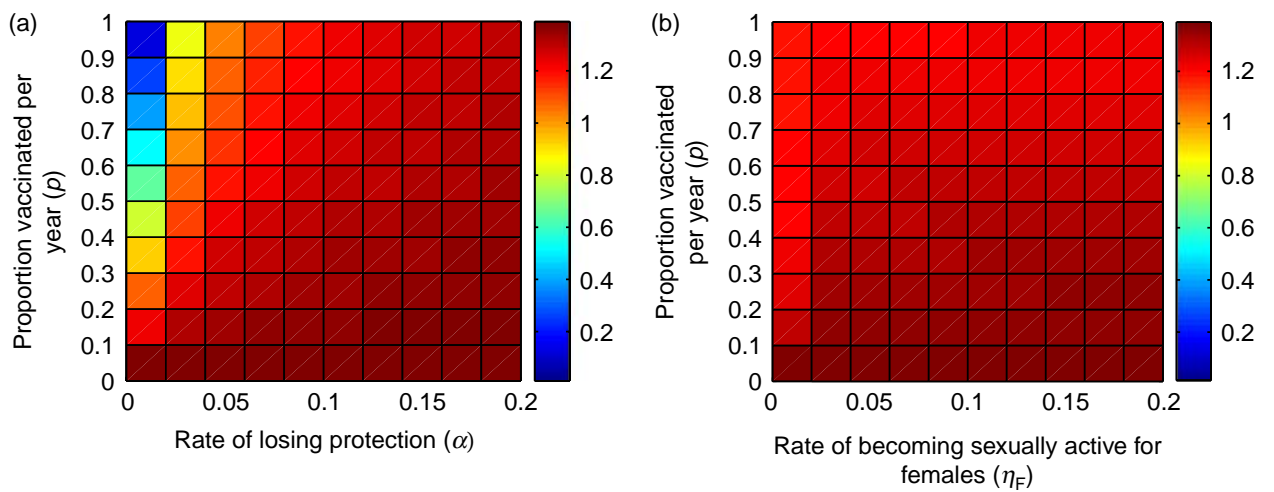

Figure 3. Two graphs showing the effect on $R_{0}^{\mathrm{e}}$ of varying two parameters. Figure 3(a) shows the change in $R_{0}^{\mathrm{e}}$ against $\alpha$ and $p$, whereas 3(b) shows $R_{0}^{\mathrm{e}}$ against $\eta_{\mathrm{F}}$ and $p$. All other parameters are standard, taken from Table B1.

\subsection{Female and male vaccination}

In Figure 5, we compare conditions (4) and (6), from which we see that the addition of male vaccination makes eradication of infection possible for a wider range of parameter
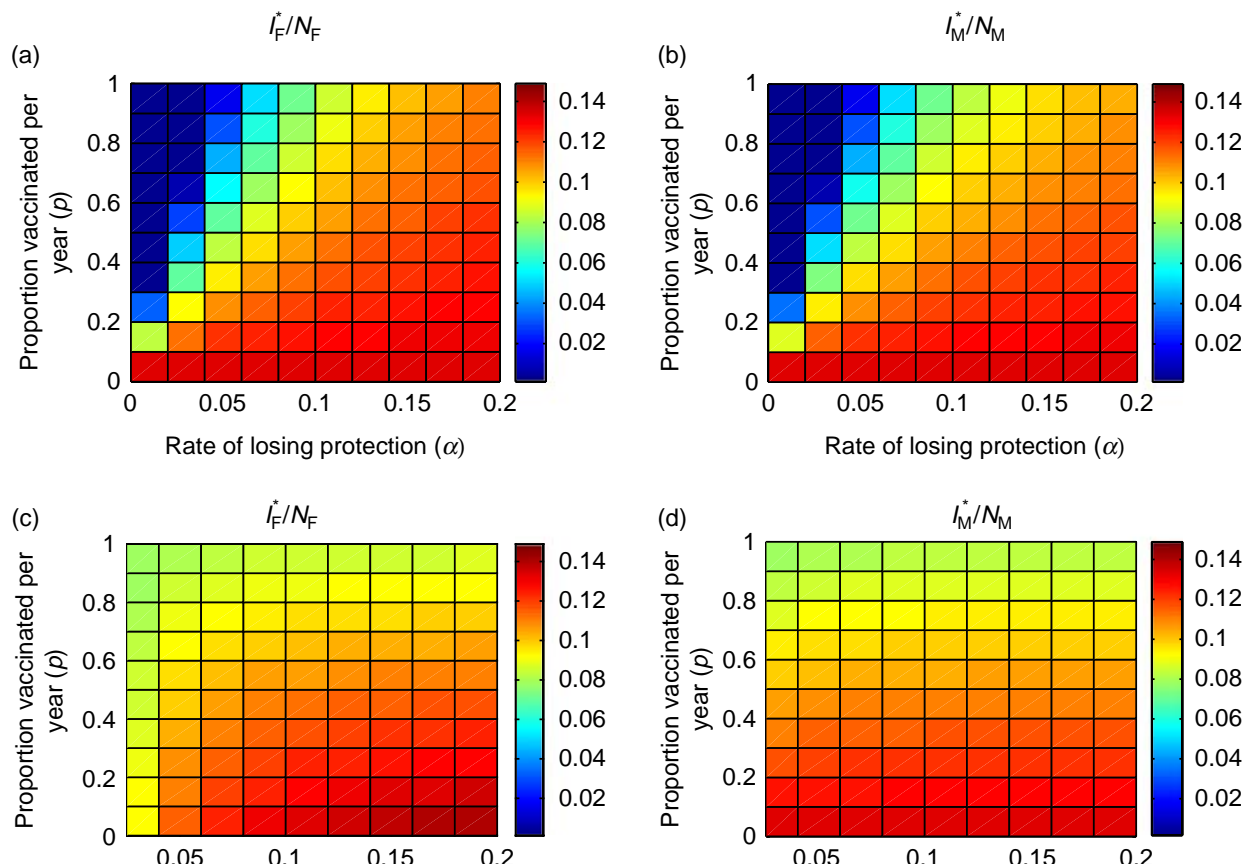

Rate of becoming sexually active for females $\left(\eta_{\mathrm{F}}\right)$

Rate of becoming sexually active for females $\left(\eta_{\mathrm{F}}\right)$

Figure 4. Four graphs showing the effect on the female- and male-infected steady states as different parameters are varied. Figures 4(a) and (b) show the female- and male-infected steady states as $\alpha$ and $p$ are varied; 4(c) and (d) shows the steady states as $\eta_{\mathrm{F}}$ and $p$ vary. All parameters come from the estimated values given in Table B1. 
(a)
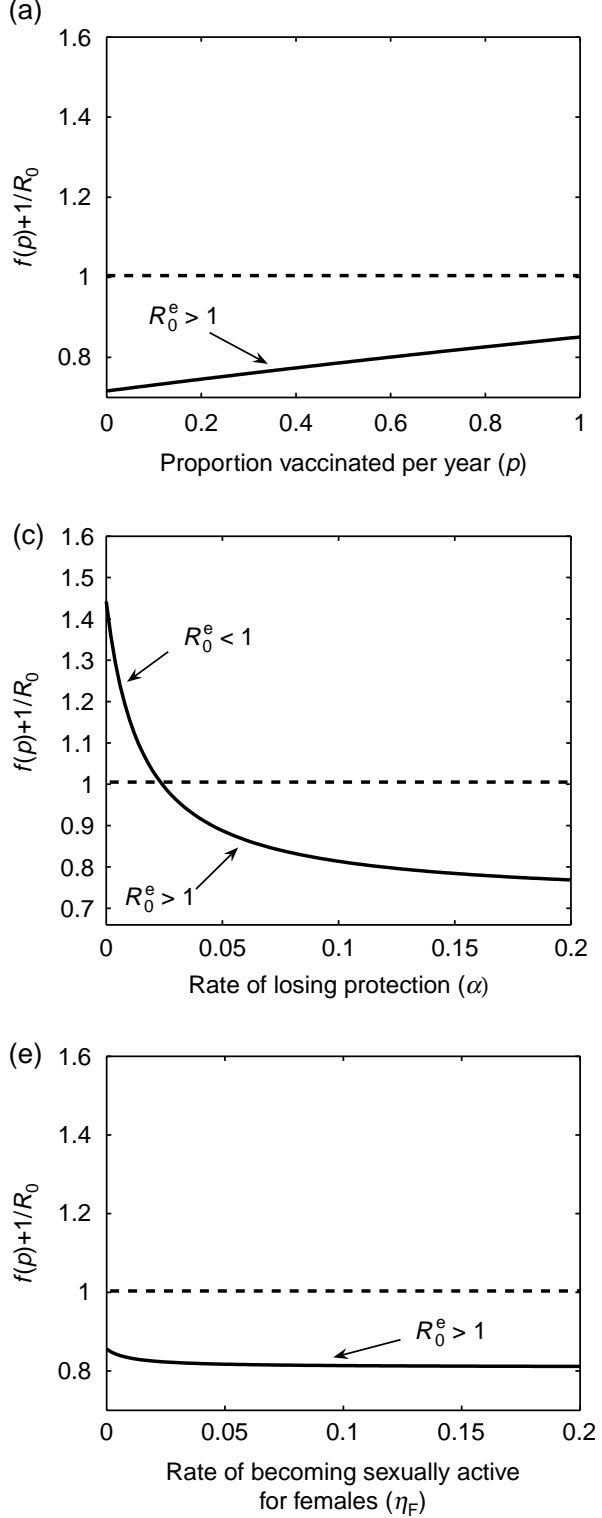

(b)
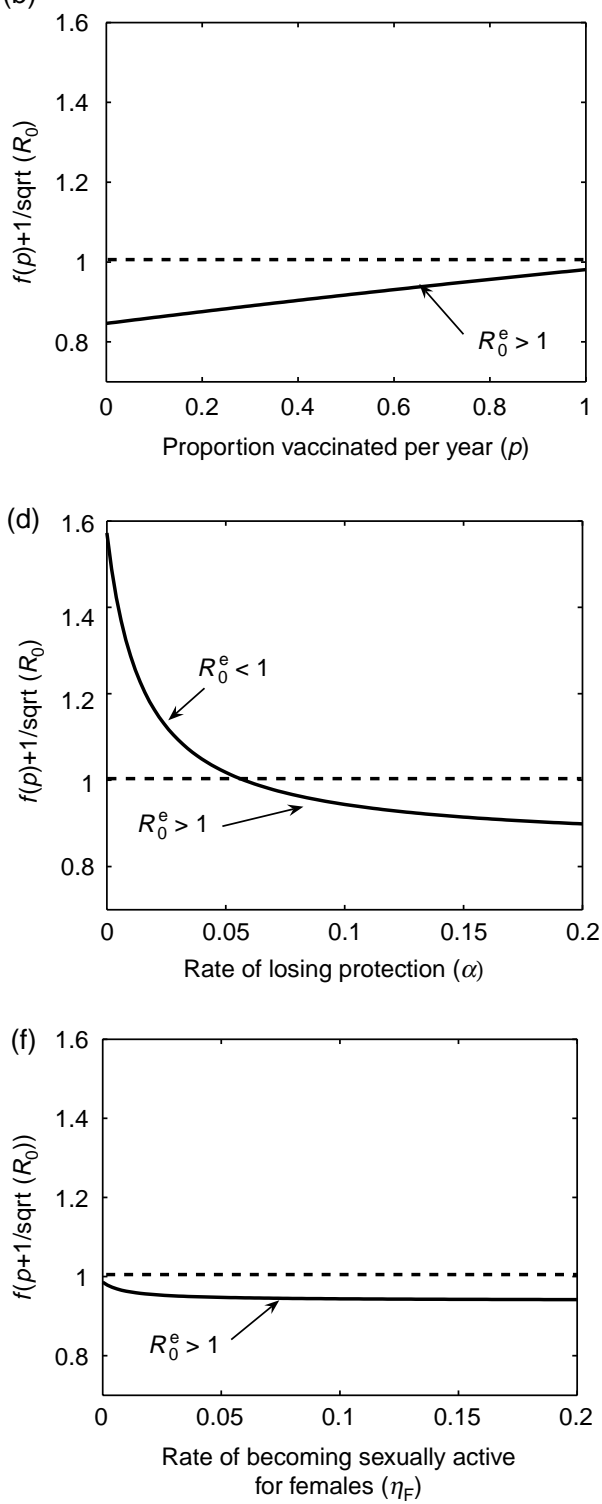

Figure 5. Graphs showing how varying different parameters affects the point at which $R_{0}^{\mathrm{e}}=1$, for the cases both with and without male vaccination. Figures 5(a), (c) and (e) show the case when there is no male vaccination. The other graphs represent the situation with male vaccination. All parameters used are given as estimates in Table B1.

values. And in the case where eradication is not possible, male vaccination allows the system to have a lower $R_{0}^{\mathrm{e}}$.

Figure 6 is analogous to Figure 4 and highlights two changes that arise with the inclusion of male vaccination:

(1) Infection prevalence in both sexes is the same since the vaccination is the same for both sexes. 
(2) There is a wider range of parameter values for which infection can be removed from the population.

To directly compare the two vaccination strategies (female-only versus both sexes), we compare values of $f(p)$ required to achieve $R_{0}^{\mathrm{e}}=1$ in (4) and (6), respectively. The proportional reduction in $f(p)$ when male vaccination is added to female-only vaccination is given as

$$
\Lambda=\frac{\left.f(p)\right|_{\text {female-only }}-\left.f(p)\right|_{\text {female and male }}}{\left.f(p)\right|_{\text {female-only }}},
$$

i.e.

$$
\Lambda=\frac{\sqrt{R_{0}}-1}{R_{0}-1} .
$$

With $R_{0} \approx 1.4$, male vaccination leads to around $45 \%$ reduction in the proportion that should be vaccinated, although the population is now doubled to $N_{\mathrm{F}}+N_{\mathrm{M}}$. This means that a greater number of individuals must be vaccinated than under female-only vaccination. In fact, (10) shows that the percentage reduction will only be greater than $50 \%$ when $R_{0}<1$.

Figure 7 shows the time profiles for each compartment of the population for the model with and without male vaccination and surface plots for the number of individuals in the
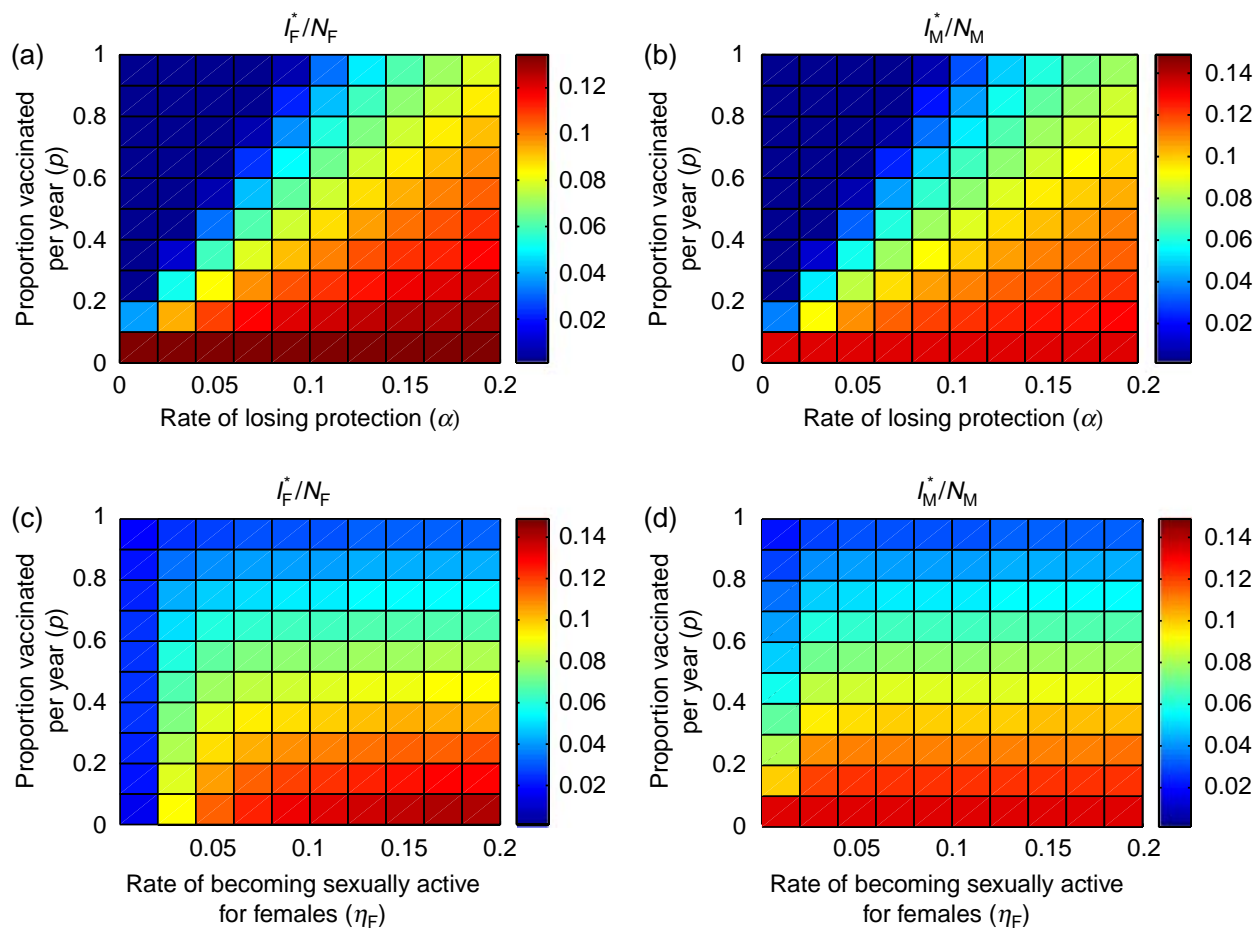

Figure 6. Four graphs showing the effect on the female- and male-infected steady states as different parameters are varied for vaccination of both sexes. Figures 6(a) and (b) show the female and male-infected steady states as $\alpha$ and $p$ are varied; 6(c) and (d) shows the steady states as $\eta_{\mathrm{F}}$ and $p$ vary. All parameters come from the estimated values given in Table B1. 

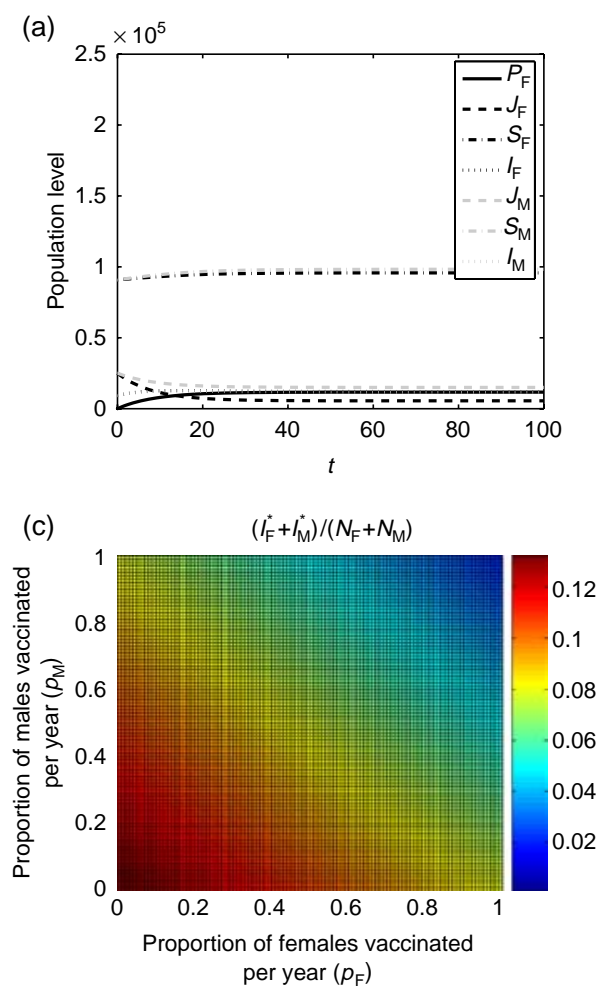
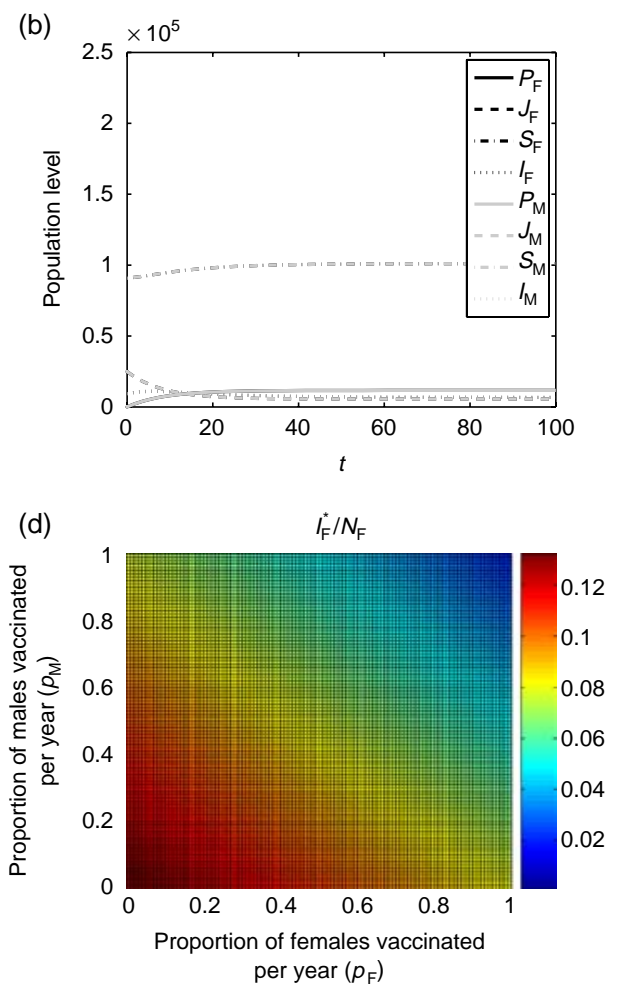

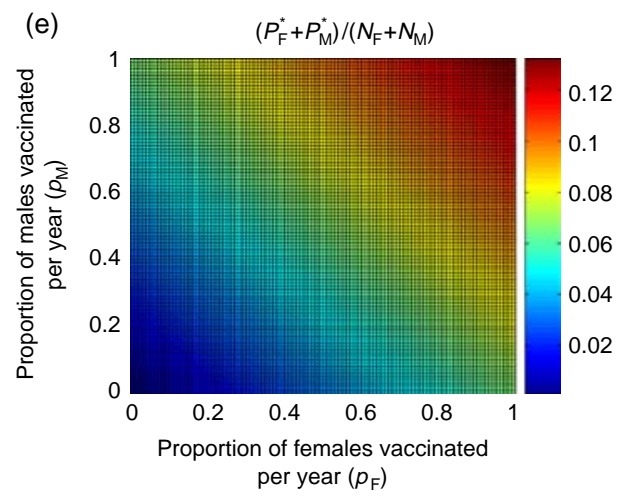

Figure 7. Graphs showing the time profiles for each of the compartments of the model without male vaccination (Figure 7(a)) and the model with male vaccination (Figure 7(b)). The second set of figures (Figure 7(c), (d), and (e)) shows the total proportions of individuals in each type of class (infected classes, infected female class and protected classes) for the model as the proportion of both female vaccinated $\left(p_{\mathrm{F}}\right)$ and male vaccinated $\left(p_{\mathrm{M}}\right)$ varies. The parameter values are all taken from the standard set as seen in Table B1.

protected and infected classes as the proportion of females vaccinated $\left(p_{\mathrm{F}}\right)$ and males vaccinated $\left(p_{\mathrm{M}}\right)$ varies. The class profiles show that male vaccination, when implemented at the same level as female vaccination, does not alter the path of the profiles. The surface plots suggest that there is a symmetry between $p_{\mathrm{F}}$ and $p_{\mathrm{M}}$, although the exact values show that there is a slight decrease in the total number of infected individuals when male 
vaccination is included, compared with female-only vaccination. It does show that it is possible to halve the value of $p$, so that the same total number of individuals are vaccinated and not see an increase in infection prevalence.

\section{Discussion}

The results of this model show that, under certain circumstances, it is possible to force $R_{0}^{\mathrm{e}}$ under 1, and thus it may be possible to eradicate HPV from the population. We assessed the effect that different behavioural and vaccination parameters have on the value of $R_{0}^{\mathrm{e}}$. Figure 2 shows that by increasing the proportion vaccinated, the value of $R_{0}^{\mathrm{e}}$ will decrease. We see the opposite effect with $\alpha$ and $\eta_{\mathrm{F}}$, as an increase in either of these parameters will cause $R_{0}^{\mathrm{e}}$ to increase. These are all as we would expect, although note that we see something close to a linear relationship between $p$ and $f(p)$, but a nonlinear relationship exists both between $\alpha$ and $f(p)$ and between $\eta_{\mathrm{F}}$ and $f(p)$. We also see that changing the value of $\alpha$ has the greatest effect on $f(p)$, suggesting that the duration of vaccine protection will have an important effect on the final outcome.

The impact of $\alpha$ is confirmed in Figure 3, where we see plots of $R_{0}^{\mathrm{e}}$ against different parameters. For a value of $\alpha=0.1$, varying $\eta_{\mathrm{F}}$ and $p$ cannot force $R_{0}^{\mathrm{e}}$ under 1 , but for a value of $\eta_{\mathrm{F}}=0.1$, varying $\alpha$ and $p$ shows that, for $\alpha<0.04$ and $p>0.7$, it is possible to push $R_{0}^{\mathrm{e}}<1$.

Figure 4 shows how the changing values of different parameters affect the infected steady states directly. This again supports the hypothesis that $\alpha$ has a much greater impact than $\eta_{\mathrm{F}}$, especially at higher values of $p$. Figures 4(a) and (b) show that, for a low value of $\alpha$, it is possible to reach the disease-free steady state (in both genders), but that the disease-free steady state cannot be reached for $\alpha=0.1$ and varying $\eta_{\mathrm{F}}$ and $p$.

Figure 5 shows how varying certain parameters can affect whether $R_{0}^{\mathrm{e}}<1$ or not, and we find that only for a long duration of protection is it possible to force $R_{0}^{\mathrm{e}}<1$. However, the duration of protection needed for this to happen is decreased when male vaccination is included, suggesting that male vaccination may come into play if the vaccination is shown to last less than 40 years.

Figure 6 shows the plots of infection prevalence (as in Figure 4), but for a model containing both male and female vaccinations. As expected, including vaccination on both sexes increases the range of values of $\alpha$ and $\eta_{\mathrm{F}}$ for which prevalence is very low, although this occurs for a wider range of values when varying $\alpha$ and $p$ than when varying $\eta_{\mathrm{F}}$ and $p$ - which matches the results gained from the case of female-only vaccination. We also saw that the inclusion of male vaccination (at the same rate as for females) led to a decrease of $45 \%$ in the percentage of individuals that needed to be vaccinated. However, as these individuals came from both the male and female classes, this actually leads to an increase in the total number of individuals that need to be vaccinated. We found that it would only be possible to vaccinate fewer individuals when $R_{0}<1$ and vaccination may not be appropriate in this case anyway.

Figure 7 shows the benefit of including male vaccination. Including male vaccination would not increase the number of infected individuals; the exact values indicate that vaccinating the same number of individuals (both males and females) as would be vaccinated in the female-only vaccination strategy actually leads to a slight decrease in the total prevalence of infection at steady state. This is positive in itself, but holds further benefits if HPV becomes recognized as a significant factor in other genital cancers.

These results show the importance of considering a range of different factors when assessing the potential success of the vaccine. We see that the most important vaccination 
parameter of those we considered was $\alpha$. From the behavioural parameters considered, it is clear that the value of $z$ will also have an important effect, as we saw it was possible to force $R_{0}^{\mathrm{e}}<1$ by varying $z$, where the other behavioural parameters (e.g. the rate at which individuals become sexually active) could not.

The inclusion of a sexually non-active class is a key difference between this and other papers. Coupled with the inclusion of waning immunity, having this class allows us to explicitly consider the effect of vaccination for an STI in a group that is (largely) not yet sexually active. We also consider male vaccination and the effect this had. While this is not new [5,21], we did not consider this from a cost-effective point of view, so could look solely at the impact male vaccination had on values such as $R_{0}^{\mathrm{e}}$.

The importance of interplay between vaccination, epidemiological and behavioural parameters is clearly demonstrated here. Of these, epidemiological parameters are essentially fixed, but vaccination parameters may be adjusted via public health strategy (p) and further pharmaceutical developments $(\alpha)$. Modifying behavioural parameters $\left(j_{i}, \eta_{i}, z\right)$ to assist the effectiveness of the vaccination programme is a more challenging problem, which might only be addressed by educational programmes. Whether the significance of altering behaviours can be made clear through such a programme remains to be seen; the linkage between HPV and cervical cancer might just be the motivating connection that convinces the public.

\section{Acknowledgements}

The authors would like to acknowledge clinician Dushyant Mital, Milton Keynes General Hospital, for introducing us to interesting problems related to HPV vaccination policy. The authors would also like to acknowledge the use of the NATSAL II [19] data in calculating some of the parameters. Copyright for these data belongs to National Centre for Social Research and A. Johnson, K. Fenton, A. Copas, C. Mercer, A. McCadden, C. Carder, G. Ridgway, K. Wellings, W. Macdowall and K. Nanchahal, and the data are held at the UK Data Archive. The copyright holders and the UK Data Archive bear no responsibility for further analysis or interpretation of the data. The authors acknowledge the use of the Omnibus Survey Report No. 30, Contraception and Sexual Health, 2005/06. This report is covered by Crown Copyright.

\section{Conflict of Interest}

The authors have no conflict of interest. No local ethical approval was required for this paper.

\section{Funding}

V. L. Brown is supported by EPSRC funding. K. A. Jane White was supported in part by a Leverhulme Research Fellowship.

\section{References}

[1] M. Adams, B. Jasani, and A. Fiander, Human Papillomavirus (HPV) prophylactic vaccination: Challenges for public health and implications for screening, Vaccine 25 (2007), pp. 3007-3013.

[2] J.M. Agosti and S.J. Goldie, Introducing HPV vaccine in developing countries - key challenges and issues, New Engl. J. Med. 356 (2007), pp. 1908-1910.

[3] R. Anderson and R. May, Infectious Diseases of Humans, Oxford University Press, Oxford, 1992.

[4] M. Arbyn and J. Dillner, Review of current knowledge on HPV vaccination: An appendix to the European guidelines for quality assurance in cervical cancer screening, J. Clin. Virol. 38 (2007), pp. 189-197. 
[5] R.V. Barnabas, P. Laukkanen, P. Koskela, O. Kontula, M. Lehtinen, and G.P. Garnett, Epidemiology of HPV 16 and cervical cancer in Finland and the potential impact of vaccination: Mathematical modelling analyses, PLoS Med. 3 (2006), p. e138.

[6] BBC, Life expectancy to soar. Available at http://news.bbc.co.uk/1/hi/health/1977733.stm (2002).

[7] BBC, Cancer jab plea for girls aged 11. Available at http://news.bbc.co.uk/1/hi/health/ 5411038.stm (2006).

[8] BBC, Many parents 'block cancer jab'. Available at http://news.bbc.co.uk/1/hi/health/ 7365613.stm (2008).

[9] N.F. Britton, Essential Mathematical Biology, Springer, Berlin, 2003.

[10] A.N. Burchell, R.L. Winer, S. de Sanjose, and E.L. Franco, Chapter 6: Epidemiology and transmission dynamics of genital HPV infection, Vaccine 24(Suppl. 3) (2006), pp. 52-61.

[11] K.S. Cuschieri, H.A. Cubie, M.W. Whitley, A.L. Seagar, M.J. Arends, C. Moore, G. Gilkisson, and E. McGoogan, Multiple high risk HPV infections are common in cervical neoplasia and young women in a cervical screening population, J. Clin. Pathol. 57 (2004), pp. 68-72.

[12] Department of Health News Distribution Services, HPV vaccine recommended for NHS immunisation programme. Available at http://nds.coi.gov.uk/environment/fullDetail.asp? ReleaseID=325799\&NewsAreaID=2 (2007).

[13] E.H. Elbasha, Global stability of equilibria in a two-sex HPV vaccination model, Bull. Math. Biol. 70 (2008), pp. 894-909.

[14] E.H. Elbasha and A.P. Galvani, Vaccination against multiple HPV types, Math. Biosci. 197 (2005), pp. 88-117.

[15] M. Kohli, N. Ferko, A. Martin, E.L. Franco, D. Jenkins, S. Gallivan, C. Sherlaw-Johnson, and M. Drummond, Estimating the long-term impact of a prophylactic human papillomavirus 16/18 vaccine on the burden of cervical cancer in the UK, Br. J. Cancer 96 (2007), pp. $143-150$.

[16] G.R. Leggatt and I.H. Frazer, HPV vaccines: The beginning of the end for cervical cancer, Curr. Opin. Immunol. 19 (2007), pp. 232-238.

[17] M. Markman, Human papillomavirus vaccines to prevent cervical cancer, The Lancet 369 (2007), pp. 1837-1839.

[18] A.R. McLean and S.M. Blower, Modelling HIV vaccination, Trends Microbiol. 3 (1995), pp. $458-463$.

[19] National Centre for Social Research, National Survey of Sexual Attitudes and Lifestyles II, 2000-2001 [computer file], Colchester, Essex: UK Data Archive [distributor] (2005), sN: 5223.

[20] J. Peto, C. Gilham, J. Deacon, C. Taylor, C. Evans, W. Binns, M. Haywood, N. Elanko, D. Coleman, R. Yule, and M. Desai, Cervical HPV infection and neoplasia in a large population-based prospective study: The Manchester cohort, Br. J. Cancer 91 (2004), pp. $942-953$.

[21] A.V. Taira, C.P. Neukermans, and G.D. Sanders, Evaluating human papillomavirus vaccination programmes, Emerg. Infect. Dis. 10 (2004), pp. 1915-1922.

[22] T. Taylor, L. Keyse, and A. Bryant, Omnibus Survey Report No. 30, Contraception and Sexual Health, 2005/06, Office for National Statistics (2006).

[23] H. Trottier and E.L. Franco, The epidemiology of genital human papillomavirus infection, Vaccine 24(Suppl. 1) (2006), pp. 1908-1910.

[24] T.C. Wright, F.X. Bosch, E.L. Franco, J. Cuzick, J.T. Schiller, G.P. Garnett, and A. Meheus, Chapter 30: HPV vaccines and screening in the prevention of cervical cancer; conclusions from a 2006 workshop of international experts, Vaccine 24(Suppl. 3) (2006), pp. 251-261. 


\section{Appendix A: Model equations}

The model used in the report is as follows:

$$
\begin{gathered}
\frac{\mathrm{d} P_{\mathrm{F}}}{\mathrm{d} t}=p \frac{N_{\mathrm{F}}}{\phi}-\left(\alpha+\frac{1}{\phi}\right) P_{\mathrm{F}}, \\
\frac{\mathrm{d} J_{\mathrm{F}}}{\mathrm{d} t}=(1-p) j_{\mathrm{F}} \frac{N_{\mathrm{F}}}{\phi}-\left(\eta_{\mathrm{F}}+\frac{1}{\phi}\right) J_{\mathrm{F}}+q \alpha P_{\mathrm{F}}, \\
\frac{\mathrm{d} S_{\mathrm{F}}}{\mathrm{d} t}=\left(1-j_{\mathrm{F}}\right)(1-p) \frac{N_{\mathrm{F}}}{\phi}+(1-q) \alpha P_{\mathrm{F}}+\eta_{\mathrm{F}} J_{\mathrm{F}}-\frac{z \beta}{N_{\mathrm{M}}-J_{\mathrm{M}}} I_{\mathrm{M}} S_{\mathrm{F}}+\gamma_{\mathrm{F}} I_{\mathrm{F}}-\frac{1}{\phi} S_{\mathrm{F}}, \\
\frac{\mathrm{d} I_{\mathrm{F}}}{\mathrm{d} t}=\frac{z \beta}{N_{\mathrm{M}}-J_{\mathrm{M}}} I_{\mathrm{M}} S_{\mathrm{F}}-\left(\gamma_{\mathrm{F}}+\frac{1}{\phi}\right) I_{\mathrm{F}}, \\
\frac{\mathrm{d} J_{\mathrm{M}}}{\mathrm{d} t}=j_{\mathrm{M}} \frac{N_{\mathrm{M}}}{\phi}-\left(\eta_{\mathrm{M}}+\frac{1}{\phi}\right) J_{\mathrm{M}}, \\
\frac{\mathrm{d} S_{\mathrm{M}}}{\mathrm{d} t}=\left(1-j_{\mathrm{M}}\right) \frac{N_{\mathrm{M}}}{\phi}+\eta_{\mathrm{M}} J_{\mathrm{M}}-\frac{z \beta}{N_{\mathrm{F}}-J_{\mathrm{F}}} I_{\mathrm{F}} S_{\mathrm{M}}+\gamma_{\mathrm{M}} I_{\mathrm{M}}-\frac{1}{\phi} S_{\mathrm{M}}, \\
\frac{\mathrm{d} I_{\mathrm{M}}}{\mathrm{d} t}=\frac{z \beta}{N_{\mathrm{F}}-J_{\mathrm{F}}} I_{\mathrm{F}} S_{\mathrm{M}}-\left(\gamma_{\mathrm{M}}+\frac{1}{\phi}\right) I_{\mathrm{M}} .
\end{gathered}
$$

Where $P_{\mathrm{F}}$ is the female protected class, $J_{i}$ the juvenile or non-sexually active class, $S_{i}$ the sexually active or susceptible class and $I_{i}$ the infected class (where $i=\mathrm{F}, \mathrm{M}$, female or male). All parameters are as given in the main article and solution of steady states leads to $R_{0}^{\mathrm{e}}$ as discussed in the text.

The initial conditions were chosen as a proportion of the total (gender-specific) population, but we do specify that $P_{\mathrm{F}}(0)=0$. We note, however, that the model outcomes discussed in this paper do not depend on the choice of initial conditions.

\section{Appendix B: Steady states}

We can find the steady states of the system, which are

$$
\begin{gathered}
P_{\mathrm{F}}^{*}=\frac{p N_{\mathrm{F}}}{\phi(\alpha+1 / \phi)}, \\
J_{\mathrm{F}}^{*}=\frac{(1-p)(\alpha+1 / \phi) j_{\mathrm{F}} N_{\mathrm{F}}+q \alpha p N_{\mathrm{F}}}{\phi\left(\eta_{\mathrm{F}}+1 / \phi\right)(\alpha+1 / \phi)},
\end{gathered}
$$

and

$$
J_{\mathrm{M}}^{*}=\frac{j_{\mathrm{M}} N_{\mathrm{M}}}{\phi\left(\eta_{\mathrm{M}}+1 / \phi\right)},
$$

$I_{\mathrm{F}}^{*}=0$ or

$$
I_{\mathrm{F}}^{*}=(z \beta) \frac{\left(\left(1-\frac{1}{R_{0}}\right)\left(N_{\mathrm{F}}-J_{\mathrm{F}}^{*}\right)-P_{\mathrm{F}}^{*}\right)}{\left(z \beta+\gamma_{\mathrm{F}}+(1 / \phi)\right)},
$$

with $I_{\mathrm{M}}^{*}=0$ or

$$
I_{\mathrm{M}}^{*}=\frac{(z \beta)\left(N_{\mathrm{M}}-J_{\mathrm{M}}^{*}\right)\left(\left(1-\frac{1}{R_{0}}\right)\left(N_{\mathrm{F}}-J_{\mathrm{F}}^{*}\right)-P_{\mathrm{F}}^{*}\right)}{z \beta\left(N_{\mathrm{F}}-P_{\mathrm{F}}^{*}-J_{\mathrm{F}}^{*}\right)+\left(\gamma_{\mathrm{M}}+(1 / \phi)\right)\left(N_{\mathrm{F}}-J_{\mathrm{F}}^{*}\right)} .
$$

Substituting all other steady state values into the equations for $N_{i}$ gives the steady state values of $S_{i}\left(S_{\mathrm{F}}^{*}=N_{\mathrm{F}}^{*}-P_{\mathrm{F}}^{*}-J_{\mathrm{F}}^{*}-I_{\mathrm{F}}^{*}, S_{\mathrm{M}}^{*}=N_{\mathrm{M}}^{*}-J_{\mathrm{M}}^{*}-I_{\mathrm{M}}^{*}\right)$. 
Analysis of the full model system (which involves seven state variables) can be reduced to explore the behaviours of an equivalent two-dimensional system in the following way. The governing equations for $P_{\mathrm{F}}, J_{\mathrm{F}}$ and $J_{\mathrm{M}}$ are uncoupled from the remainder of the system, and hence stability of their steady states can also be determined separately from the full system. Since we are assuming a constant population size for each of the males and females, the dimension of the infection system $\left(S_{\mathrm{F}}, I_{\mathrm{F}}, S_{\mathrm{M}}, I_{\mathrm{M}}\right)$ can further be reduced to consider the two-dimensional system for $\left(I_{\mathrm{F}}, I_{\mathrm{M}}\right)$ :

$$
\begin{gathered}
\frac{\mathrm{d} I_{\mathrm{F}}}{\mathrm{d} t}=\frac{z \beta}{N_{\mathrm{M}}-J_{\mathrm{M}}} I_{\mathrm{M}}\left(N_{\mathrm{F}}-P_{\mathrm{F}}-J_{\mathrm{F}}-I_{\mathrm{F}}\right)-\left(\gamma_{\mathrm{F}}+\frac{1}{\phi}\right) I_{\mathrm{F}}, \\
\frac{\mathrm{d} I_{\mathrm{M}}}{\mathrm{d} t}=\frac{z \beta}{N_{\mathrm{F}}-J_{\mathrm{F}}} I_{\mathrm{F}}\left(N_{\mathrm{M}}-J_{\mathrm{M}}-I_{\mathrm{M}}\right)-\left(\gamma_{\mathrm{M}}+\frac{1}{\phi}\right) I_{\mathrm{M}},
\end{gathered}
$$

where $J_{\mathrm{F}}(t), P_{\mathrm{F}}(t)$ and $J_{\mathrm{M}}(t)$ are determined from the full system. Behaviour of the full system can then be inferred from the behaviour of this system. We calculate the stability of the disease-free steady state $\left(I_{\mathrm{F}}=I_{\mathrm{M}}=0\right)$ using the Jacobian of (15) evaluated at that point. This gives

$$
J=\left(\begin{array}{cc}
-\left(\gamma_{\mathrm{F}}+\frac{1}{\phi}\right) & \frac{z \beta\left(N_{\mathrm{F}}-P_{\mathrm{F}}^{*}-J_{\mathrm{F}}^{*}\right)}{N_{\mathrm{M}}-J_{\mathrm{M}}^{*}} \\
\frac{z \beta\left(N_{\mathrm{M}}-J_{\mathrm{M}}^{*}\right)}{N_{\mathrm{F}}-J_{\mathrm{F}}^{*}} & -\left(\gamma_{\mathrm{M}}+\frac{1}{\phi}\right)
\end{array}\right) .
$$

Stability of this steady state requires that $\operatorname{trace}(J)<0$ and $\operatorname{determinant}(J)>0$. Since $\operatorname{trace}(J)<0$ for all parameter combinations, stability of the disease-free steady state requires that

$$
\left(\gamma_{\mathrm{F}}+\frac{1}{\phi}\right)\left(\gamma_{\mathrm{M}}+\frac{1}{\phi}\right)>\frac{(z \beta)^{2}\left(N_{\mathrm{F}}-P_{\mathrm{F}}^{*}-J_{\mathrm{F}}^{*}\right)}{N_{\mathrm{F}}-J_{\mathrm{F}}^{*}}
$$

i.e.

$$
\frac{R_{0}\left(N_{\mathrm{F}}-P_{\mathrm{F}}^{*}-J_{\mathrm{F}}^{*}\right)}{N_{\mathrm{F}}-J_{\mathrm{F}}^{*}}<1
$$

\begin{tabular}{|c|c|c|c|}
\hline Parameters & Symbol & Range of values & Reference \\
\hline $\begin{array}{l}\text { Proportion of protected class not sexually } \\
\text { active when vaccine wears off }\end{array}$ & $q$ & $\begin{array}{l}0 \leq q \leq 1 \\
q \approx(0.616 \alpha+0.05) /(1+\alpha)\end{array}$ & [19] \\
\hline $\begin{array}{l}\text { Proportion of the average population in } \\
\text { one year that enters the 'juvenile' class }\end{array}$ & $j_{i}$ & $0 \leq j_{i} \leq 1, j_{i} \approx 0.9$ & {$[19]$} \\
\hline $\begin{array}{l}\text { Proportion of the average population in } \\
\text { one year that is vaccinated }\end{array}$ & $p$ & $0 \leq p \leq 1$ & N/A \\
\hline Average lifespan & $\phi$ & $64 \leq \phi \leq 69, \phi \approx 65$ & {$[6]$} \\
\hline Rate of losing protection & $\alpha$ & $0.01 \leq \alpha \leq 0.2, \alpha \approx 0.1$ & [16] \\
\hline Rate of becoming sexually active & $\eta_{i}$ & $0.005 \leq \eta_{i} \leq 0.23, \eta_{i} \approx 0.1$ & [19] \\
\hline Average duration of infection & $\gamma_{i}^{-1}$ & $0.5 \leq \gamma_{i} \leq 2, \gamma_{i} \approx 1$ & {$[10,23]$} \\
\hline $\begin{array}{l}\text { Average number of sexual partners } \\
\text { per year }\end{array}$ & $z_{i}$ & $0 \leq z_{i} \leq 2, z_{i} \approx 2$ & {$[22]$} \\
\hline $\begin{array}{l}\text { Probability of transmission of infection } \\
\text { per sexual partner }\end{array}$ & $\beta$ & $0.4 \leq \beta \leq 0.8, \beta \approx 0.6$ & {$[5]$} \\
\hline
\end{tabular}

The left-hand side of this equation is equal to

$$
R_{0}^{\mathrm{e}}=R_{0}\left(1-\frac{p\left(\eta_{\mathrm{F}}+(1 / \phi)\right)}{\phi(\alpha+1 \phi)\left(\eta_{\mathrm{F}}+(1 / \phi)\right)-\left((1-p)(\alpha+(1 / \phi)) j_{\mathrm{F}}+q \alpha p\right)}\right) .
$$

Table B1. Table of parameters. 
For $R_{0}^{\mathrm{e}}<1$, the disease-free steady state is stable and is the only steady state of the system. For $R_{0}^{\mathrm{e}}>1$, the disease-present steady state exists and the disease-free steady state is unstable.

\section{B.1 Critical values}

Corresponding to $R_{0}^{\mathrm{e}}=1$, we can derive critical values for $\alpha$ and $\eta_{\mathrm{F}}$, which are

$$
\alpha^{\text {crit }}=\frac{p(\eta+1 / \phi)-\left(1-\frac{1}{R_{0}}\right)\left(\eta+\frac{1}{\phi}-\frac{(1-p) j}{\phi}\right)}{\left(1-\frac{1}{R_{0}}\right)(\phi(\eta+1 / \phi)-(1-p) j)-q p},
$$

and

$$
\eta_{\mathrm{F}}^{\text {crit }}=\frac{\left(1-\frac{1}{R_{0}}\right)\left((1-p)(\alpha+(1 / \phi)) j_{\mathrm{F}}+q \alpha p\right)}{\phi(\alpha+(1 / \phi))\left(1-\frac{1}{R_{0}}\right)-p}-\frac{1}{\phi},
$$

respectively. Then for $R_{0}^{\mathrm{e}}<1$ we require $\alpha<\alpha^{\text {crit }}$ and $\eta_{\mathrm{F}}<\eta_{\mathrm{F}}^{\text {crit }}$, provided the denominators are positive. 


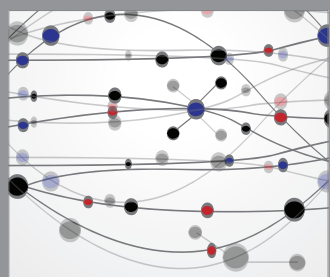

The Scientific World Journal
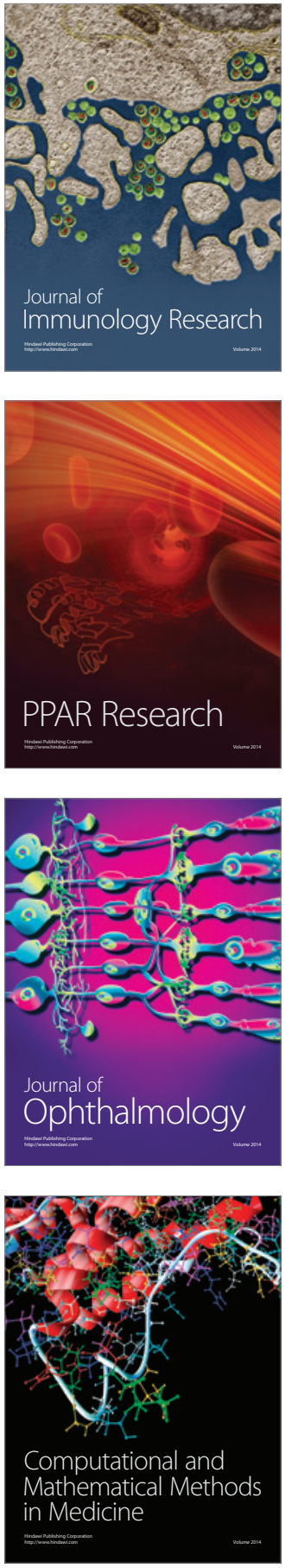

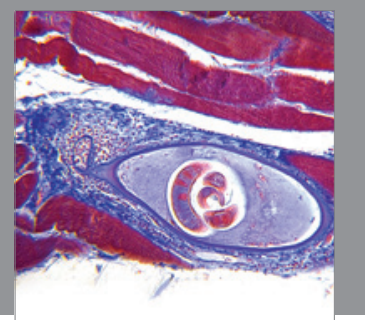

Gastroenterology

Research and Practice
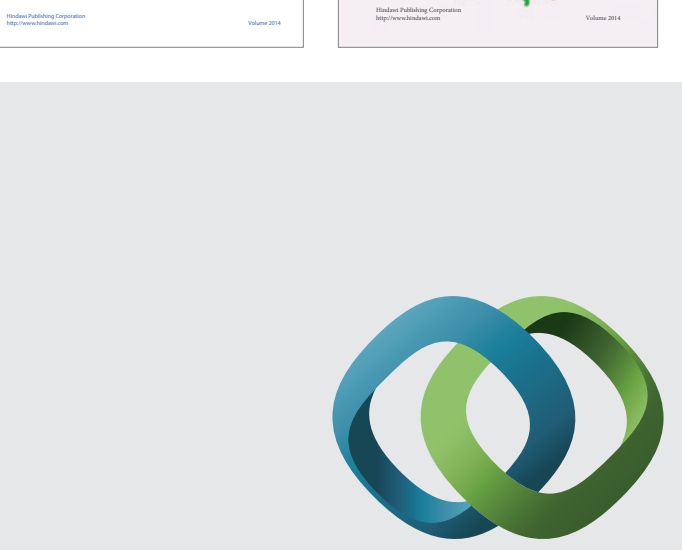

\section{Hindawi}

Submit your manuscripts at

http://www.hindawi.com
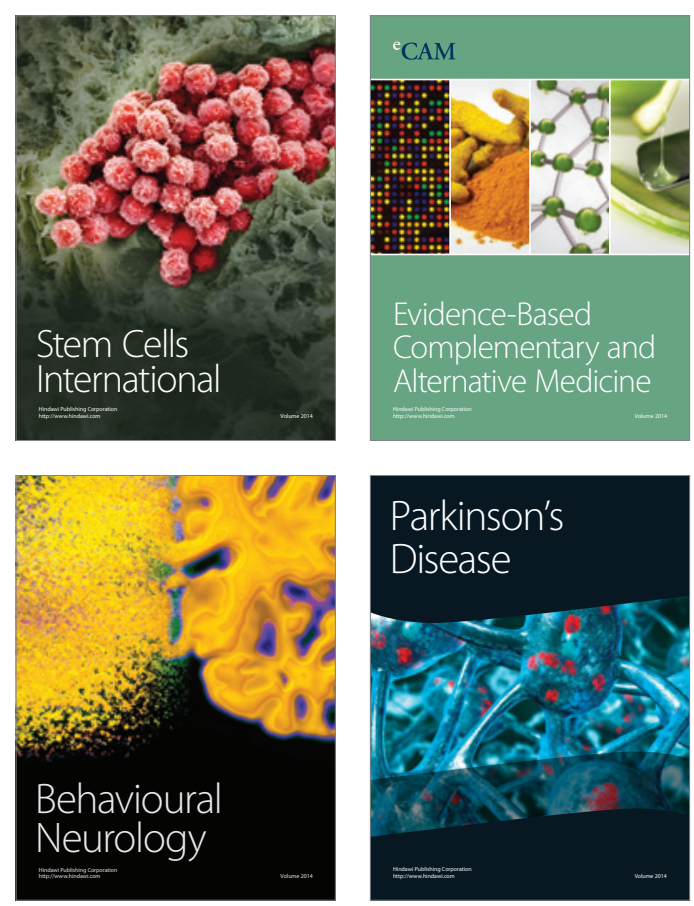

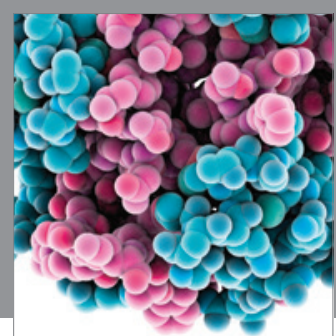

Journal of
Diabetes Research

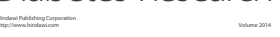

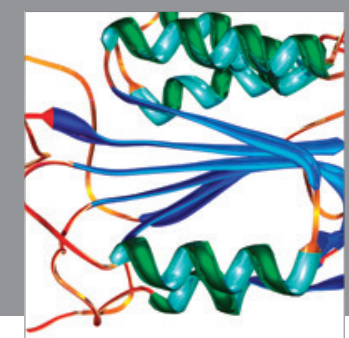

Disease Markers
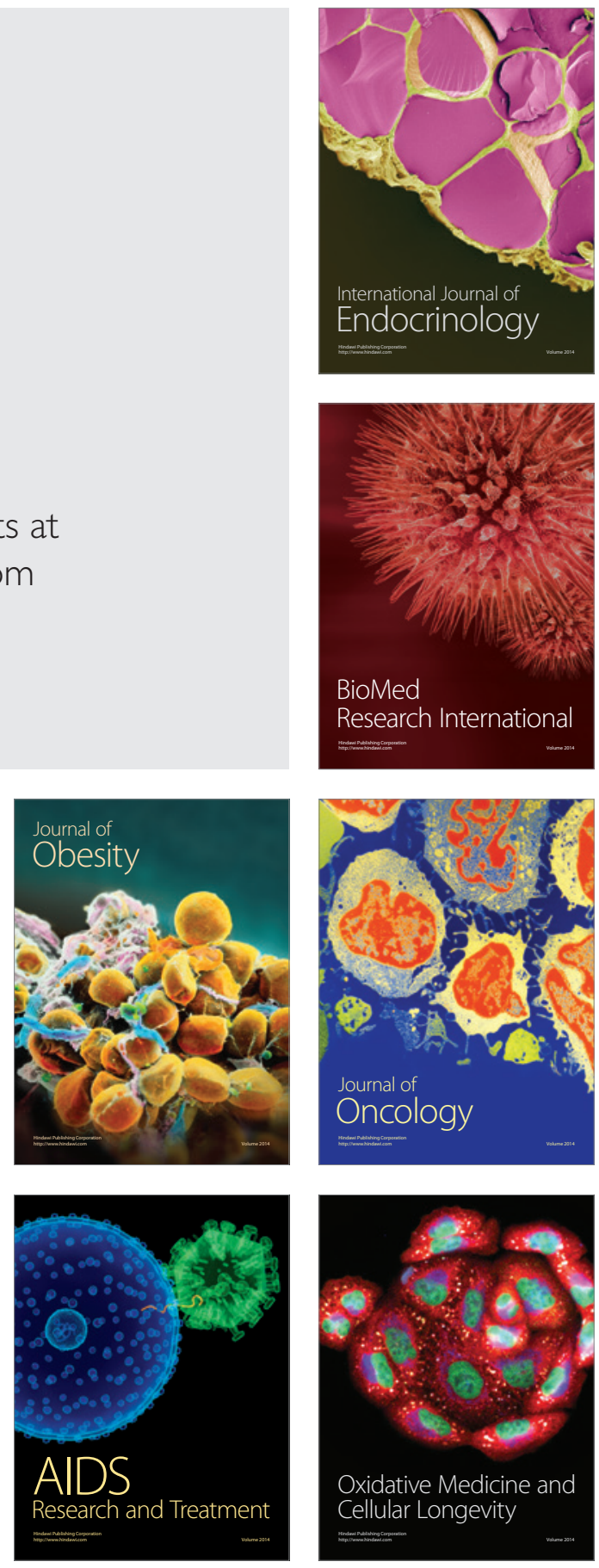